\title{
A research into phase synchronization algorithm
}

\author{
Yin Shuhua ${ }^{1, a}$ \\ ${ }^{1}$ Xi'an fanyi university, Xi'an 710105, China \\ aemail: shuhua_y@126.com
}

Keywords: Communication Receiver; Carrier Phase Synchronization; Discrete Information Maximum; likelihood Estimation

\begin{abstract}
The research studies the carrier and timing synchronization algorithms in all-digital communication receivers. According to the optimal estimation theory of carrier phase and symbol timing synchronization technology, the estimation algorithm of the maximum likelihood carrier phases is proposed and used in computer simulations. The simulation results reveal that when the signal-noise ratio (SNR) and the observation length $\mathrm{L}$ meet certain requirements, the curve is highly smooth and symmetric. The allowable minimum observation length is the capture length under this condition. If the SNR is larger than $10 \mathrm{~dB}$, as for 16QAM signals, the synchronized algorithm for maximum likelihood carrier phase can track phases within 16 symbols.
\end{abstract}

\section{Introduction}

Traditional communication receivers use coherent demodulation to receive signals. The circuits for carrier recovery utilize Costas loop or its modified forms. While the nonlinear clock extraction method is employed in timing circuits to extract the synchronized information from the demodulated baseband signals so as to perform symbol timing and control sample collectors. The partial signals in each symbol period are sent to the balancer composed of tapped delay lines to be balanced and put into the decision device afterwards. After decision decoding, transmitting code sequences are recovered ${ }^{[1]}$. The typical characteristics of this communication receiver lie in that it realizes demodulation by using simulators and generates coherent clocks and carriers by controlling the voltage controlled oscillator (VCO) after feeding back the error signals of timing and carrier phases.

In general, the communication receiving system which is composed of analogue devices shows many problems, such as large volume, high power consumption and cost, inconvenient debugging, easy to break down and hard to process intelligent signals. To solve these problems, the all-digital intelligent communication receivers come into being by integrating micro-electronic technology, digital signal processing technology, computer technology and communication technology.

The structure of all-digital communication receivers is different from that of traditional receivers. The key difference is that the former neither uses the phase-locked loop circuit nor feeds back the signals to the simulation parts for feedback control. The work processes of full digital communication receivers are displayed as follows: Clock signals with high stability are generated by an oscillator to control the direct sampling of analog-to-digital converter (ADC) for received signals. Afterwards, the sampled and quantified digital signals are sent to the digital signal processor (DSP) system to carry out digital conversion, phase matching filter, and orthogonal matching filter, successively. In this way, the symbol timing synchronization, the recovery of carrier phases and symbol decision and decoding are implemented. Therefore, carrier and timing synchronization is the key to study all-digital communication receivers.

The carrier phases and symbol timing of the all-digital communication receivers are estimated separately and jointly, which is similar to the synchronization system of analog communication receivers. This study mainly introduces the estimation algorithm of maximum likelihood carrier phases based on optimal estimation theory in separate estimation. 


\section{The optimal estimation theory}

The carrier phase and symbol timing synchronized technology based on the optimal estimation theory is demonstrated as follows: The carrier phase $\Phi$ and the transmission delay $\tau$ represent two parameters to be estimated, Then the optimal estimation of parameters is determined according to the relevant criteria. In the estimation of synchronized parameters of signals, the maximum likelihood criterion and the maximum posteriori probability criterion are commonly used ${ }^{[1-2]}$.

Maximum likelihood criterion If the density function of likelihood probability of a received signal $r(t)$ is expressed as $P(r \mid \Phi, \tau)$, the maximum likelihood estimations of $\Phi$ and $\tau$ are described as the phase $\Phi_{M L}$ and the time delay $\tau_{M L}$ when the density function of likelihood probability is the maximum.

Maximum posteriori probability criterion If the density function of posteriori probability of the received signal $r(t)$ is presented as $P(\Phi, \tau \mid r)$, the phase $\Phi_{\text {MAP }}$ and the time delay $\tau_{\text {MAP }}$ that maximize the density function of posteriori probability are the maximums a posteriori probability (MAP) estimation ${ }^{[3]}$.

In the all-digital communication receivers, the synchronized parameters $\Phi$ and $\tau$ are regarded as unknown and undetermined parameters to be estimated by the maximum likelihood criterion, namely, the maximum likelihood estimation algorithm. In this estimation algorithm, it is assumed that parameters to be estimated change slowly. The receivers are used to observe received signals for a period of time $T_{0}$ and extracted estimation values, thus this period of time $T_{0} \quad\left(T_{0}>>\right.$ $T$ (symbol period)) is defined as observation interval. The estimator produces an estimated value in each observation interval. For the fast changing parameters, Calman filter algorithm is used to realize fast tracking.

Joint maximum likelihood estimation algorithm estimates multiple parameter values simultaneously by maximizing the joint probability density function of multiple parameters. Generally speaking, better results can be obtained in joint estimation of multiple parameters than that of separate estimation of a single parameter. In other words, the estimation variance of signal parameters obtained from joint estimation is smaller than that obtained from separate estimation of each parameter. Although the synchronized algorithm of joint estimation is superior to that of separate estimation in theory, it is difficult to in implementation due to the complex circuits and large calculation load. Taking the practical application and processing abilities of the existing DSP chips into account, the synchronized algorithm of separate estimation shows simple structure, good performance and high feasibility and is easy to be realized ${ }^{[4]}$.

\section{Algorithm principles}

Equivalent low pass transmit signal for digital linear modulation technique can be defined as:

$$
s(t)=\sum_{n=0}^{\infty} a_{n} g_{t}(t-n T)
$$

Where, $\left\{a_{n}\right\}, T$ and $g_{t}(t)$ represent discrete information (complex value) symbol sequence, symbol period and shaping impulse, respectively. Suppose that an amplitude attenuation factor $\rho(t)$, a phase offset $\theta(t)$ and a time delay $\varepsilon(t) T$ of channels are introduced through channels. According to the all-digital implementation scheme, the local carriers vibrate at the fixed frequency, and the phase shift caused by the frequency deviation is shown on the phase offset and affected by noise. The equivalent low-pass form of received signals is expressed as:

$$
r(t)=\sum_{n=-\infty}^{\infty} \rho(t) a_{n} g_{t}(t-n T-\varepsilon(t) T) e^{j \theta(t)}+n(t)
$$

Where, $T, n(t)$ and $n_{0}$ indicate known symbol period, white Gaussian noise and the unilateral power spectrum density, respectively. The estimation of carrier phases is to determine the value of 
$\theta(t)$. In the actual system, the AGC circuit is used to compensate or maintain the attenuation factor $\rho(t)$ at a constant value $\rho$, while the changes of $\varepsilon(t)$ and $\theta(t)$ are slower compared with symbol rate. The above condition is easy to be met in each section (the observation length is $L$ symbols), so $\varepsilon(t)$ and $\theta(t)$ are regarded as constants $\varepsilon$ and $\theta$, respectively. In this way, the problem of timing recovery is converted to estimating the timing constant $\varepsilon$ and phase constant $\theta$, and updating section by section. If the impulse response of the receiving filter is $g_{r}(t)$, the received signals are shown as following after filtering:

$$
\tilde{r}(t)=r(t) * g_{r}(t)
$$

It is assumed that $g(t)=g_{t}(t) * g_{r}(t)$, where, * represents the convolution. Under proper timing conditions, after sampling by using the filters, Formula (2) can be expressed as:

$$
\tilde{r}_{n}=\sum_{n=-\infty}^{\infty} \rho a_{n} g(n T) e^{j \theta_{n}}+\tilde{n}_{n}=\rho a_{n} e^{j \theta_{n}}+\tilde{n}_{n}
$$

Where, $g(n T)=1$ represents the sampling value of shaping impulse meeting the conditions without crosstalk of sampling points, and $\theta_{n}$ is carrier phase to be estimated. Because the values of $\theta_{n}$ are periodic, it is selected from $[-\pi, \pi)$ and discussed in an observation length $\mathrm{L}$. According to the requirements of precision and calculation of phase retrieve, the interval $[-\pi, \pi)$ is quantified as $N_{\theta}$ grades, and the value $\theta$ is obtained from $N_{\theta}$ discrete values. When $\theta$ is valued as $\theta_{m}$, the density function of joint likelihood probability for the sampling sequence of $\mathrm{L}$ received symbols is presented as:

$$
P\left(\vec{r} \mid \theta_{m}, \vec{a}\right)=\left(\prod_{n=0}^{L-1} 2 \pi \sigma^{2}\right)^{-1} \times \exp \left(-\frac{1}{2 \sigma^{2}} \sum_{n=0}^{L-1}\left|\tilde{r}_{n}-\rho a_{n} e^{j \theta_{m}}\right|^{2}\right)
$$

$\theta_{m}$ that maximizes Formula (5) is the maximum likelihood estimation of the carrier phases. The corresponding logarithm likelihood function can be deduced from Formula (5):

$$
\Lambda\left(\theta_{m}, \vec{a}\right)=-\sum_{n=0}^{L-1}\left|\tilde{r}_{n}-\rho a_{n} e^{j \theta_{m}}\right|^{2}
$$

Similarly, the phase estimation obtained by maximizing Formula (6) is the maximum likelihood estimation of the carrier phase. The calculation of this formula is related to the transmitted symbol sequence $\left\{a_{n}\right\}$ and this formula is calculated through traversing $\mathrm{M}$ possible stars in the constellation diagram, with respect to the unknown transmitted symbols. For example, as to 16QAM, $\mathrm{M}=16$, for each test value $\theta_{m}$ of $\theta$ in each received symbol, the likelihood probabilities of 16 possible transmitted symbols need to be calculated.

In order to reduce the test times and calculation, when the transmitted sequence is unknown, Formula (6) is modified as follows:

$$
\Lambda\left(\theta_{m}, \vec{a}=\vec{a}_{o p t}\right)=-\sum_{n=0}^{L-1}\left|\frac{1}{\rho} \tilde{r}_{n} e^{-j \theta_{m}}-a_{n_{o p t}}\right|^{2}
$$

The mean square distance is defined as:

$$
V\left(\theta_{m}, \vec{a}=\vec{a}_{o p t}\right)=-\Lambda\left(\theta_{m}, \vec{a}=a_{o p t}\right)=\sum_{n=0}^{L-1}\left|\frac{1}{\rho} \tilde{r}_{n} e^{-j \theta_{m}}-a_{n_{o p t}}\right|^{2}
$$

Where, $a_{n_{\text {opt }}}$ represents the most probable star in the constellation diagram when symbol phase $\mathrm{n}$ is $\theta_{m}$. In other words, with respect to the most probable star showing the least Euclidean distance with the obtained signal points, the likelihood probability is larger than that of other stars. For any unknown symbols and test phases, there is undoubtedly a most probable star correspondingly. Therefore, as long as the likelihood probability corresponding to the most probable phase is calculated for one time, the maximum likelihood probability of the test phase values can be 
obtained.

\section{Algorithm simulation}

In order to test the correctness of the synchronized algorithm for maximum likelihood carriers, simulation is carried out by using MATLAB software under different conditions. The information source is randomly generated 16QAM sequence with cosine signals applied as the shaping filter and the quantified interval of phases being $90^{\circ} / 64$. Furthermore, the radian of preset phase $31 \pi / 64$ is used to simulate the actual phase. According to Formula (8), the corresponding mean square distance is calculated, and the test phase corresponding to the minimum value is selected.

The feasibility of the synchronized algorithm for the maximum likelihood carrier phases of 16QAM modulation signal is validated applying the observation length L, SNR, and preset phase as parameters ${ }^{[5]}$.

The relationship between observation length $L$ and mean square distance When the roll-off factor $\alpha$ and SNR are 0.5 and $9 \mathrm{~dB}$, respectively, the distributions of the observation length and mean square distance with quantization phases are simulated. The simulation results are shown in Fig. 1. It can be seen from Fig. 1 that there are minimum values in the curves of mean square distance under three conditions, and the minimum values of test phases correspond to the maximum likelihood estimations of the carrier phases. When the phase is preset as $31 \pi / 64$ in the simulation program, the valley point is found in the $31^{\text {st }}$ test phase. In addition, it reveals that although differences are shown in the mean square distance under different conditions, these differences do not affect the identification for the minimum values under the same condition ${ }^{[6]}$.

The relationship between SNR and mean square distance As the roll-off factor $\alpha$ is 0.5 and the observation length is represented by 16 symbols, the simulated distributions of different SNRs and mean square distances with quantified phases are displayed in Fig. 2.
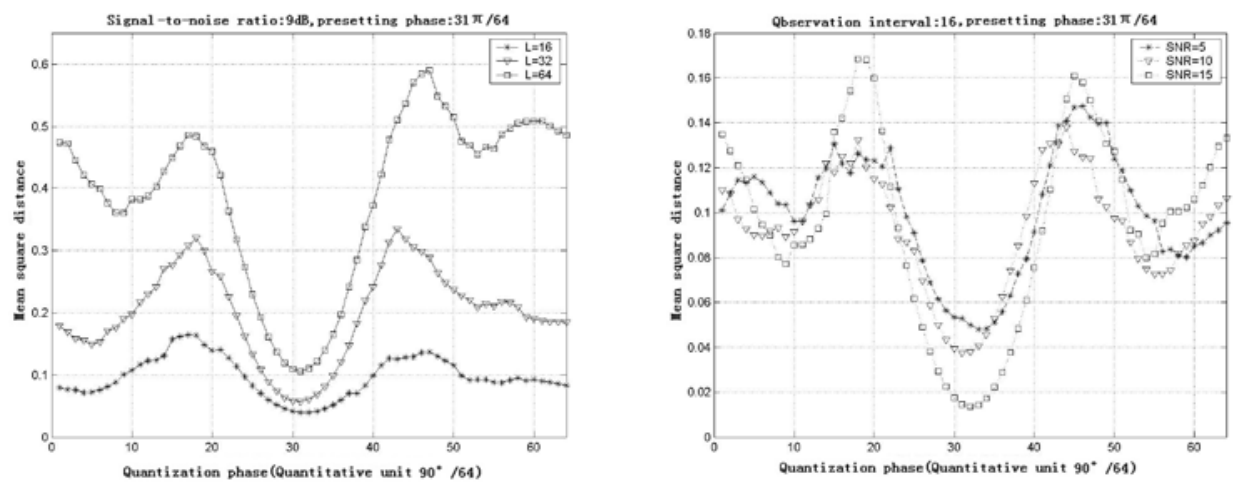

Fig. 1 The relationship between observation length $L$ and mean square distance

Fig. 2 The relationship between SNR and mean square distance

As demonstrated in the figure, when SNR is $5 \mathrm{~dB}$, a few errors are shown in phase correction, while phases can be corrected in other conditions. The height of the main lobes reflects the accuracy of the phase estimation, and the phase corresponding to the minimum value is the maximum likelihood estimation.

The relationship between the preset phase and mean square distance When the observation length and SNR are 16 symbols and $9 \mathrm{~dB}$, respectively, the relationship between the preset phase and mean square distance is displayed in Fig. 3. 


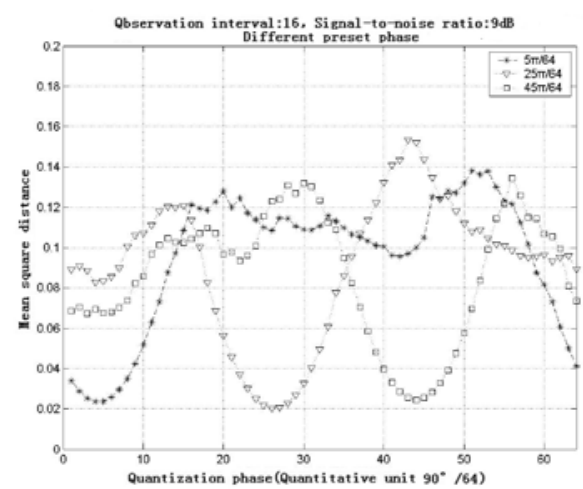

Fig. 3 The relationship between the preset phase and mean square distance

As presented in Fig. 3, when the SNR and observation length are the fixed values, the accuracy of phase estimation is basically same, which indicates that different phase errors can be corrected by using this algorithm. Moreover, the relative height of main lobes is basically same, showing the same difficulty for realizing phase acquisition.

\section{Conclusions}

It is concluded according to the simulation results that the curve presents good smoothness and symmetry when the SNR and the observed length L satisfy certain conditions. This result shows that this algorithm presents good convergence. The allowable minimum observation length is considered as the capture length under this condition. In addition, as for 16QAM signal, the synchronized algorithm for maximum likelihood carrier phase tracks phases within 16 symbols when the SNR is larger than $10 \mathrm{~dB}$. Apart from these, when the observation length is fixed, the larger the SNR is, the higher the relative height of main lobes and thereby the easier the capture. While if the SNR is a constant, the larger the observation length L, the higher the accuracy of phase estimation is. This algorithm with simple structure is easy to realize in DSP as it merely works in time domain. Through practical application, this algorithm has been used in the demodulation of 16QAM satellite signals with small carrier frequency in the MATLAB software.

\section{Acknowledgement}

In this paper, the research was sponsored by the science research projects of education department in shaanxi province government (Project No. 16JK2078).

\section{References}

[1] WU lifu, WANG Hua,CHENG Yi,et al. An improved algorithm for blind estimation of reverberation time based onmaximum likelihood [J].Journal of Applied Acoustics, 2015,35 (4):288-294.

[2] XU Feng,QIU lede,Wang Yu. Fine carrier phase recovery method for APSK signals[J].Journal of Chongqing University of Posts and Telecommunications(Natural Science Edition). 2013, 25(2): 281-284.

[3] ZHOU xiangchao,XUE RUI,ZHAO danfeng,et al. Soft carrier phase synchronization algorithm for continuousphase modulations signals[J].Journal of Jilin University(Engineering and Technology Edition), 2015,45(1):291-296.

[4] ZHOU Cheng,HUANG gaoming,DAN hongchang,et al. Bias compensation algorithm based on maximum likelihood estimation for passive localization using TDOA and FDOA measurements[J].Acta Aeronautica et Astronautica Sinice, 2014, 35(3): 979-986.

[5] ZHAO danfeng,ZHOU xiangchao,FU Fang. Code-Aided Carrier Phase Synchronization Algorithmfor LDPC-Coded Systems[J].Journal of Astrunautics, 2013, 34(5): 699-705.

[6] LIU anbang,AN jianping,WANG aihua. A Fast and Accurate BPSK Carrier Recovery Algorithm[J]. Transactions of Beijing institute of Technology, 2011,31(7): 823-827. 
